\title{
Stress state effect on mechanical behaviour of shape memory alloys: Experimental characterisation and modelling
}

\author{
L. Orgéas and D. Favier
}

\author{
Laboratoire Sols-Solides-Structures, UMR 5521 du CNRS, UJF-INPG, BP. 53, 38041 Grenoble \\ cedex, France
}

\begin{abstract}
As engineering components utilising the beneficial properties of shape memory alloys (SMA) become geometrically complex and as applications involve combinations of loading states, a full evaluation of the effects of stress state on stress-strain response of these materials becomes critical for the success of these applications. Such evaluation is required to establish reliable constitutive relationship to model the complex thermomechanical behaviour of SMAs.

This paper is intended to present a non-exhaustive overview on studies and results dealing with experimental characterisation and modelling of the effects of stress state on mechanical behaviour of shape memory alloys. In that respect, our presentation is mainly focused on one hand on experimental results on superelastic and ferroelastic deformation of polycrystalline alloys and on the other hand on the modelling at the macroscopic level, yielding critical reorientation or transformation constitutive equations.
\end{abstract}

\section{INTRODUCTION}

Shape memory alloys (SMAs) are known to exhibit a range of novel thermomechanical properties due to thermoelastic martensitic transformations. The superelasticity is a quasi-elastic deformation far beyond the conventional elastic limit of the material when deformed at certain temperature above $A_{f}$, the finishing temperature of the reverse martensite-to-austenite transformation. This phenomenon is associated with stress-induced martensitic (SIM) transformation. If the temperature is lowered to below $M_{f}$ the finishing temperature of the forward austenite-to-martensite transformation, the deformation proceeds by martensite reorientation. Martensite may deformed by reorientation forth and back with alternating stresses in opposite directions. This deformation mode is known as the ferroelasticity, in recognition of its phenomenological similarity to ferromagnetism. The one-way shape memory effect is observed when a specimen deformed by martensite reorientation reverts to its original shape upon heating to above $A_{f}$ Numerous experimental works have been performed in the past three decades aiming at characterising the thermo-mechanical behaviour in order to provide experimental evidences for the establishment of constitutive models. These experimental studies have been devoted to both single-crystal and polycrystal specimens, mainly using Cu-based and NiTi SMAs. Most mechanical testing has been carried out in tension using wire specimens. Less work has been done to characterise properties under other stress states. As engineering components become geometrically complex and as applications involve combinations of loading states, it becomes important to characterise material properties in different stress states. This will allow the establishment of reliable constitutive equations to model the complex thermomechanical behaviour of SMAs.

The present paper is intended to give a non-exhaustive overview on the studies and results dealing with experimental characterisation and modelling of the influence of stress state on mechanical behaviour of shape memory alloys. The goal of such studies is to apply the finite element method to calculate the mechanical behaviour of engineering components fabricated from SMAs. In that respect, our presentation is mainly focused on one hand on the experimental results on superelastic and ferroelastic deformation of polycrystalline alloys and on the other hand on the macroscopic modelling that leads to the establishment of constitutive equations. 


\section{EXPERIMENTAL STUDIES USING UNIAXIAL TENSION}

\subsection{Nominal stress-nominal strain curves}

Conventionally, most mechanical testing of SMAs has been carried out in uniaxial tension using wires or straight specimens cut from sheets [1-9], shapes that are the most commonly accessible forms for SMAs. Figure 1 shows typical engineering tensile stress-strain curves [7, 10] for the ferroelastic behaviour (a) and superelastic behaviour (b) of polycrystalline NiTi alloys. The ferroelastic behaviour was measured at $295 \mathrm{~K}$ using a wire specimen of $1.6 \mathrm{~mm}$ in diameter. The specimen had a nominal composition of $\mathrm{Ti}-$ 50.0 at $\% \mathrm{Ni}$ and was annealed at $983 \mathrm{~K}$. The superelastic behaviour was measured at $333 \mathrm{~K}, 348 \mathrm{~K}, 358 \mathrm{~K}$ and $363 \mathrm{~K}$ using wire specimens of $1 \times 1 \mathrm{~mm}^{2}$. The specimen had a nominal composition of Ti-50.2at\% Ni and was annealed at $723 \mathrm{~K}$. In most of published results, the tensile stress $\sigma$ is estimated as the engineering stress $F / S_{o}$ ( $F$ and $S_{o}$ being respectively the current applied force and the initial cross-section) whereas the tensile strain $\varepsilon$ is estimated as the engineering strain $\Delta l / l_{o}\left(l_{o}\right.$ and $\Delta l$ being respectively the initial gauge length and its variation). It is usually stated that initial stress causes only elastic distortion of martensite or austenite lattice in ferroelasticity or in superelasticity, respectively, until reaching a threshold tensile stress at which a strong decrease in the slope of the stress-strain curves is observed (horizontal plateau in Figure 1(a) and (b)). The threshold stress is interpreted as the stress required to induce martensite reorientation $\left(\sigma_{m}\right)$ or martensitic tranformation $\left(\sigma_{t r}\right)$ for the two situations presented. In superelasticity, the transformation stress $\sigma_{t r}$ is observed to depend on temperature $T$ following a linear Clausius-Clapeyron relation $[11,2]$ (as discussed later and illustrated in Figure 5(b)).

(a)

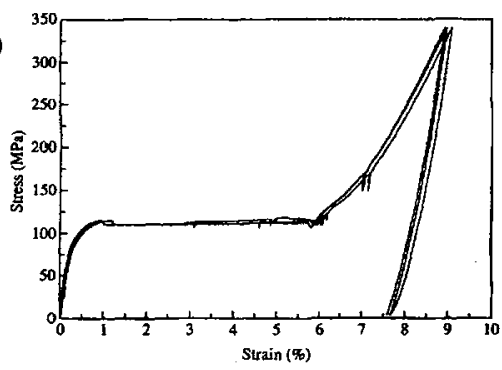

(b)

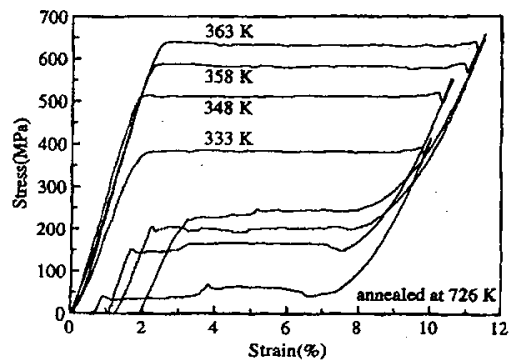

Figure 1: Tensile stress-strain curves obtained with NiTi polycrystals (a) in ferroelasticity [7];(b) in superelasticity [10].

\subsection{Non-homogeneity of the tensile test}

Superelastic tensile tests in most of $\mathrm{NiTi}$ alloys exhibit Lüders type engineering stress plateau [8], with an upper yield stress during loading and an inverse stress peak during unloading, as shown in Figure 1(b). This localised deformation has been studied by in-situ optical observations [8] and more recently by in situ full-field temperature monitoring and by local deformation measurement using multiple miniature extensometers [9]. The onset and the propagation of the localised deformation have been interpreted as being associated with the nucleation and propagation of the martensitic transformation. However, as underlined by Liu [12], several aspects impose a challenge to this nucleation-propagation hypothesis. First, a uniform temperature increase prior to the onset of the Luiders-like deformation [9] demonstrates that the transformation starts prior to the localisation. Second, this Lüders-like behaviour is not observed in compression or shear tests of single-crystals or polycristalline NiTi [13-16], as shown in Figure 3(b) and Figure 5(a), or in tension-compression tests of Cu based SMAs [17-19], as shown in Figure 3(a). In tension tests of NiTi SMAs, this localisation is hindered when using bulk specimens [20]. Moreover, Liu et al [7] have shown that Lüders-like behaviour could also be recorded during martensite reorientation of $\mathrm{NiTi}$ polycrystals in tension. The three almost identical tensile ferroelastic stress-strain curves plotted in Figure 1(a) are deduced from measurements of three extensometers fixed at different locations along the wire specimen (Figure 2(a)). The evolutions of the three strain measurements with time (Figure 2(b)) confirm that martensite reorientation can also lead to strain localisation and propagation phenomena.

All these remarks underline the difficulties to draw conclusions only from results of tensile tests of SMAs exhibiting Lüders-like behaviour. These difficulties are even greater in analyses dealing with partial transformations. Such analyses are often crucial in the development of reliable constitutive models suitable for many engineering applications, especially under cyclic loading conditions. Therefore, it is 
sometimes inappropriate, or misleading, to attempt to build models and constitutive relationships based only on such experimental results. In that respect, tests using other stress states than tension give the opportunity to verify common assumptions, e.g., that "the stress-strain curve has a negative slope during the transformation" and that "internal hysteresis loops are explained by means of the diagonal line" [21]. It is worth noting that the observed localisation in tension can be well simulated by finite elements method assuming either a negative [22] or a slightly positive [23] slope of the true stress-strain tensile curve. With this last assumption, no localisation is predicted in compression or shear, as observed experimentally [23].

(a)

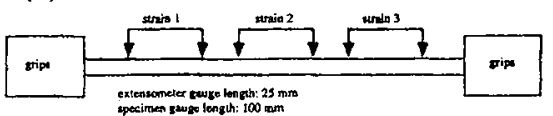

speciname gaveo lenguh $100 \mathrm{mam}$

Figure 2 : (a) Schematic illustration of tensile testing using a NiTi wire and 3 local extensometers fixed, (b) time evolutions of the 3 extensometers during the tensile test plotted in Figure 1(a) [7]. (b)

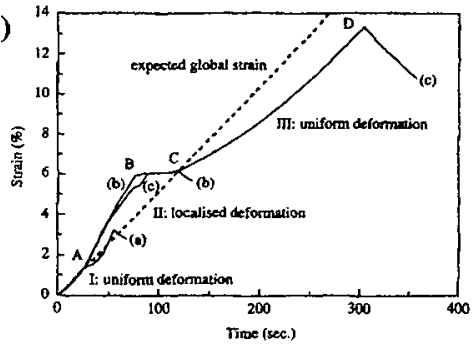

\section{EXPERIMENTAL STUDIES USING OTHER HOMOGENEOUS TESTS}

\subsection{Comparison between compression and tension}

The influence of the direction of a uniaxial normal stress (tension $v s$. compression) on superelastic stressinduced martensitic transformation has been observed on SMA single crystals as well as on textured or non-textured SMA polycrystals [6,13-14,17,19,24-32]. As an example, Figure 3 displays engineering stress-strain curves for a cyclic superelastic tension-compression test performed on one polycrystalline CuZnAl (Figure 3(a), [19]) and for two monotonic tension and compression tests performed at the same testing temperature on two identical NiTi polycrystalline plate samples (Figure 3(b), [14]). For the experiments shown in Figure 3(b), the initial isotropy of the material was confirmed by microhardness tests and several shear tests at different orientations relative to the rolling direction of the plate. Critical stress for stress-induced martensitic transformation is higher in compression than in tension for both the CuZnAl alloy shown in Figure 3(a) and for the NiTi alloy shown Figure 3(b).

(a) 40

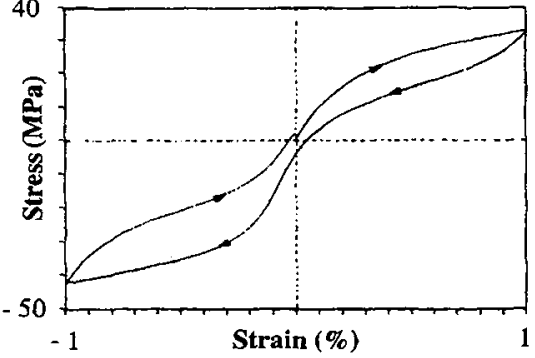

(b)

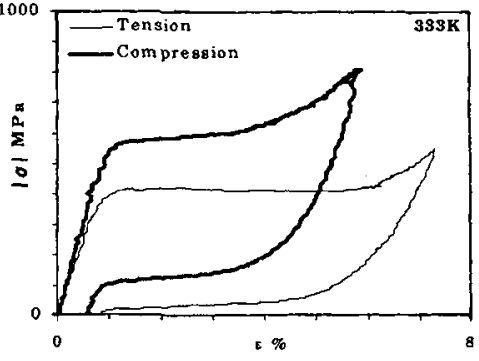

Figure 3 : Cyclic tension-compression superelastic test on one CuZnAl polycrystalline sample [19], (b) monotonic tension and compression superelastic tests on two NiTi polycrystalline samples [14].

This asymmetry is analysed more closely in Figure 4 for a cyclic tension-compression test conducted at room temperature on a CuZnAl SMA tube at low strain values [17]. The specimen exhibited critical transformation temperatures of $M_{s}=298 \mathrm{~K}, M_{f}=283 \mathrm{~K}, A_{s}=289 \mathrm{~K}$ and $A_{f}=303 \mathrm{~K}$, and had an inner diameter of $27 \mathrm{~mm}$ and an outer diameter of $30 \mathrm{~mm}$. Figure 4(b) shows the true stress-strain curve, with the strain being measured by strain gages bonded to the specimen gauge section. The evolution of the slope of the stress-strain curve is plotted as function of the strain in the vicinities of the maximum strains attained in compression (Figure 4(a)) and in tension (Figure 4(c)). These curves illustrate that there does not exist a stage after reversion during which the slope is constant. This observation defies the common assumption of a stage of elastic unloading after each reversion of stress. This leads to difficulties to estimate accurately moduli of elasticity from mechanical measurements [10]. For example, Figure 4(a) and (c) show that slopes of the stress-strain curve in the initial "elastic" region are measured to reduce by approximately one third after $0.1 \%$ strain. Similar results are published by Liu [10]. It is evident in Figure 
4(b) that the true stress is higher in compression than in tension. The modulus of elasticity, measured as the maximum stress-strain slopes just after the reversion points at $\pm 0.6 \%$, is determined to be $E_{c}=31000 \mathrm{MPa}$ for compression (Figure 4(a)) and $E_{t}=25000 \mathrm{MPa}$ for tension (Figure 4(c)). By recording circumferential strains, Poisson ratios are determined at $\pm 0.6 \%$ strain to be $\nu_{c}=0.485$ for compression and $v_{t}=0.475$ for tension [17].

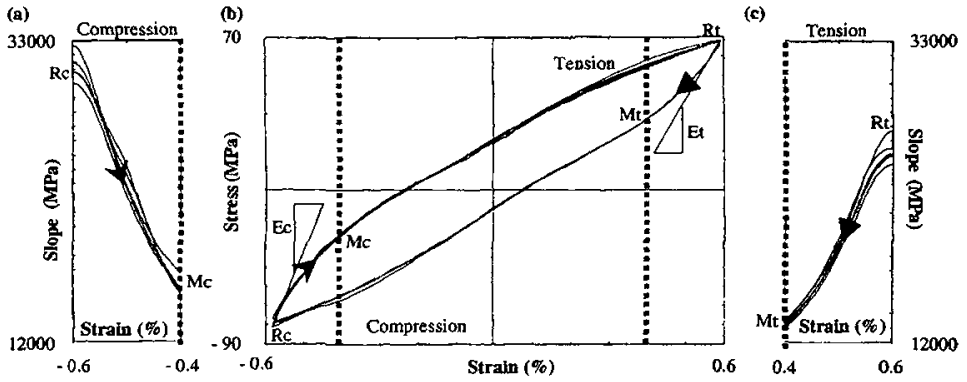

Figure 4 : Cyclic tension-compression test on a CuZnAl polycrystal : (b) stress-strain curve, (a) slope during unloading in compression, (c) slope during unloading in tension [17].

\subsection{Comparison between torsion/shear and tension/compression}

Adler et al [33] have published experimental results of tension and torsion testing of NiTi. The tensile test was performed in air using cylindrical samples of $6.35 \mathrm{~mm}$ gauge diameter. The torsion test was performed in agitated liquid using tube samples of $6.1 \mathrm{~mm}$ outer diameter and $4.0 \mathrm{~mm}$ inner diameter. Since the walls of these samples were thick, only averaged shear strain $\gamma_{\text {ave }}$ and stress $\tau_{\text {ave }}$ were estimated. Von Mises equivalent stress and strain were calculated according to $\sigma_{\text {Mises }}=\sigma_{\text {iension }}=\sqrt{3} \tau_{\text {ave }}$ and $\varepsilon_{\text {Mises }}=\varepsilon_{\text {tension }}=\gamma_{\text {ave }} / \sqrt{3}$. They concluded that equivalent stress-strain curves deduced from tension and torsion tests agreed rather well when the samples were deformed in ferroelasticity or in plasticity. The comparison was not successful for superelastic tests due to non-identical thermal testing conditions. The quasi-adiabatic tensile test led to a temperature rise of $14 \mathrm{~K}$ when the torsion tests were almost isothermal. This points out the necessity to carefully control testing temperature when samples are deformed in superelasticity.

Manach and Favier [15] have performed superelastic tension and shear tests at low strain rates on NiTi plate specimens of $1 \mathrm{~mm}$ in thickness in a liquid bath, which allows accurate control of testing temperature. They have shown that shear tests were homogeneous and neither exhibited Lüders-like deformation. The authors concluded that superelastic tension and shear compared rather well using Von Mises equivalent stress and strain.

The influence of temperature has been studied by Orgéas and Favier [14] for tension, compression and shear tests at temperatures above $\mathbf{M}_{s}$ (the testing temperature being reached by cooling the specimen). As an example, Figure 5(a) shows superelastic cyclic shear stress-strain curves at three testing temperatures above $A_{f}$. Cyclic shear tests between two opposite strains on isotropic samples lead to symmetric shear stress-strain curves. The linear dependencies of the characteristic stresses for transformation on temperature are rather well verified, as shown in Figure 5(b). The slopes of the linear relationships are determined to be $5.3,8.4$ and $12 \mathrm{MPa}^{-1}$ over a large temperature range for shear, tension and compression, respectively.

Figure 6 shows results of a cyclic torsion test performed at room temperature using identical $\mathrm{CuZnAl}$ samples as those shown in Figure 4. Figure 6(a) represents shear stress-strain curve, with the shear strain being measured by strain gages bonded to the specimen gauge section. The shear stress is calculated from the recorded torque, since the thickness of the tube is rather small compared to the average radius [18]. The evolutions of the slope of the shear stress-strain curve during the first $0.4 \%$ shear strain variation on the unloading branches after each reversion are plotted in Figure 6(b). At first, comparison of stress levels attained in tension (Figure 4(b)) and in torsion (Figure 6(a)) shows that tension and shear on the CuZnAl alloy compare rather well using Von Mises equivalent stress and strain. At an equivalent strain $\varepsilon_{\text {Mises }}$ of $0.6 \%$, the stresses measured at point Rt of Figure 4(b) and at point 7 of Figure 6(a) lead both to a Von Mises stress $\sigma_{\text {Mises }}$ of $70 \mathrm{MPa}$. This conclusion is similar to that obtained by Manach [15] comparing 
superelastic shear and tension tests on a NiTi alloy. However, the equivalent stress at the same strain of $0.6 \%$ in compression (point Rc of Figure $4(\mathrm{~b})$ ) is equal to $80 \mathrm{MPa}$, which invalidates the use of Von Mises equivalent for SMAs. Figure 6(b) illustrates that, similarly to the case of tension-compression, there does not exist a stage after the reversion during which the slope is constant. For example, the slope is reduced from $12000 \mathrm{MPa}$ at reversion point 13 to $7000 \mathrm{MPa}$ at point $\mathrm{Ms}$ after a shear strain variation of $0.4 \%$. Figure 6(b) also shows that the shear modulus of elasticity, taken as the maximum value of the slope after each reversion point is dependent on the strain. This observation agrees with previous studies by Tourabi et al [18] and by Liu and Xiang [10]. In linear isotropic elasticity, the moduli of elasticity in compression, tension and shear are related by $E_{c}=E_{s}=2 \mu(1+v)$. For an equivalent strain of $0.6 \%$ in the shear test (point 7 and 14 of Figure 6), the shear modulus is measured to be $9250 \pm 250 \mathrm{MPa}$. For the equivalent strain of $0.6 \%$ in the tension-compression test (point $\mathrm{Rc}$ and $\mathrm{Rt}$ of Figure 4), the shear moduli estimated using the above equation and the experimentally measured values of $E_{c}$ and $v_{c}$ or $E_{t}$ and $v_{t}$ are $10400 \mathrm{MPa}$ and $8500 \mathrm{MPa}$, respectively. It is seen that these values agree well with the directly measured shear modulus.

(a)

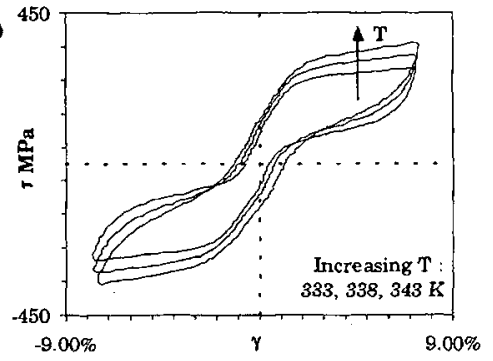

(b)

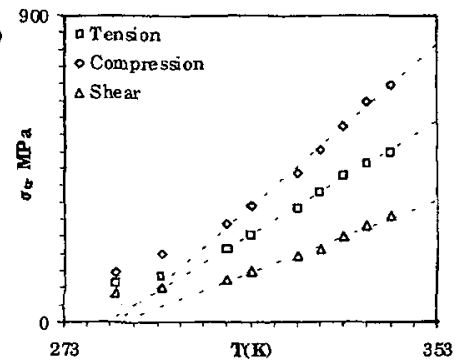

Figure 5 : (a) Cyclic superelastic shear tests performed at different temperatures on a NiTi polycrystal, (b) temperature dependence of the threshold stress to induce martensite for the same alloy in tension, compression and shear [14].
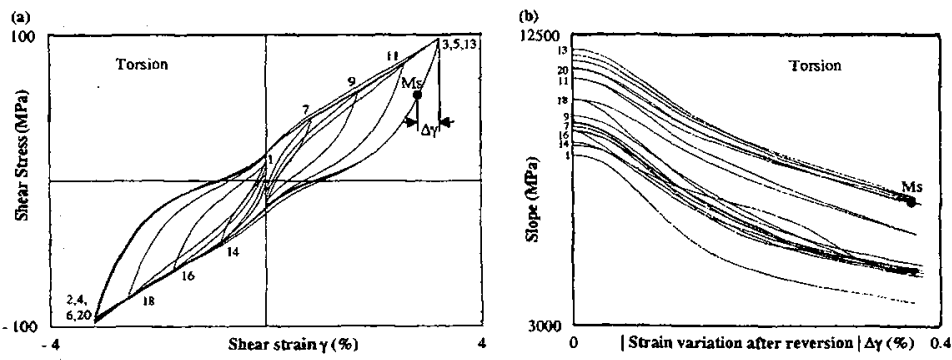

Figure 6 : Cyclic torsion test with subloops on the same CuZnAl polycrystal as that shown in Figure 4 [17], (a) stress-strain curve, (b) slope during unloading after reversion (all subloops).

\subsection{Combined loading}

In order to improve description of the criterion for 3D yield by transformation, stress-induced martensitic transformation was also investigated under multi-axial stress state [26-28,34-35].

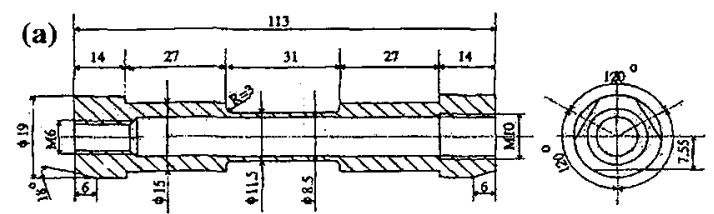

Figure 7: Combined tension-compression-torsion test on superelastic CuZnAl polycrystal : (a) shape of the tested specimen, (b) evolution of the threshold stress as a function of the stress-state in a normal

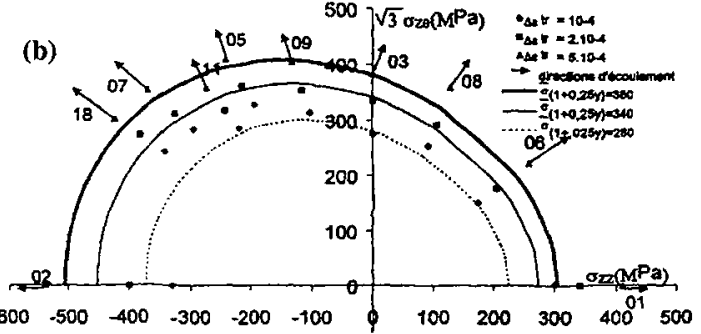
stress-shear stress diagram [26].

Lexcellent et al [26] performed combined tension-compression-torsion experiments using NiTi polycrystalline tubular samples as sketched in Figure 7(a). Deduced experimental transformation stresses 
are plotted in Figure 7 (b) in a [axial stress- $\sqrt{3} \times$ shear stress] graph. Von Mises criterion predicts that all experimental points should be located on a circle in this graph, which is obviously not verified. Lexcellent et al [26] also performed biaxial compression on cubic polycrystalline $\mathrm{CuAlBe}$ samples under superelastic deformation conditions using a unique testing apparatus. Once again, identical observations were obtained : the Von Mises criterion failed in predicting the yield transformation surface.

A further understanding of stress-induced martensitic transformation was obtained on textured NiTi and $\mathrm{CuZnAl}$ polycrystals deformed during revolution triaxial tests [27-28]. Results indicate that stressinduced martensitic transformation in textured SMAs is dependent on the applied hydrostatic pressure. In the case of $\mathrm{NiTi}$, despite the negative volume change associated with the forward transformation, the effective stress for the onset of the transformation is found to decrease with decreasing hydrostatic pressure.

\section{MODELLING}

All experimental results presented above involve radial stress states for which the stress tensor $\underline{\underline{\sigma}}$ is written in an euclidian frame $\underline{e}_{i}$ as $\underline{\underline{\sigma}}=\sigma(t) \beta_{i j} \underline{e}_{i} \otimes \underline{e}_{j}$, where $\sigma$ is a time-dependent scalar function and where $\beta_{i j}$ are scalars equal to 1 or 0 depending on the stress state. This section is devoted to modelling at macroscopic level of the stress surface delimiting the onset of transformation/reorientation for radial stress states in SMA polycrystals. In a formalism similar to the theory of plasticity [36], such criteria are defined by scalar functions $f$ depending on the components of $\underline{\underline{\sigma}}$. Transformation/reorientation are induced if $f(\underline{\underline{\sigma}})=0 . f$ relates the stress-state effects on transformation/reorientation onset.

\subsection{Role of "micro"-mechanisms at the martensite-variant level and of "meso"-structure on macroscopic SMA deformation}

The form of $f$ is strongly affected both by micro-mechanisms occurring during transformation and reorientation at the martensite-variant level and by the "meso"-structure of considered SMAs. First, stress-induced martensitic transformation as well as martensite reorientation involve very small volume change [37]. Second, many polycrystalline SMAs exhibit crystallographic textures induced by their manufacturing processes, e.g., wire drawing, rolling, etc. This causes mechanical anisotropy of textured polycristalline SMAs [43-45] due to anisotropy of superelastic deformation of SMA single crystals [3841]. Third, superelastic SMAs single crystals display asymmetric tension-compression deformation behaviour [29-30,16]. This contributes to the asymmetric tension-compression deformation behaviour observed on isotropic as well as textured polycrystalline SMAs [32, 43, 47].

Several micro-macro models have been developed to investigate the role of both textures and single crystals behaviour on macroscopic anisotropy and tension-compression asymmetry in polycrystalline SMAs [31,46-47]. Most of these models are based on the Phenomenological Theory of Martensite Crystallography (PTMC) and on self-consistent schemes [46-47]. For the present, none of them uses the more realistic micro-models developed recently [44,16]. Prediction of micro-macro models gives useful information to build macroscopic constitutive relationships. The published micro-macro models succeed in describing anisotropic superelastic behaviour of textured SMAs and asymmetric superelastic behaviour of texture-free polycrystalline $\mathrm{CuZnAl}$. However, they fail in predicting the tension-compression asymmetry observed during superelastic deformation of isotropic polycrystalline NiTi [47]. At last, to the authors' knowledge, no micro-macro model has yet been developed to model martensite reorientation. To date, most of the macroscopic constitutive relationships are thus based on experimental results.

\subsection{Macroscopic transformation/reorientation criteria}

No macroscopic form of $f$ has been proposed to account for all experimental results given previously. The question is yet to be answered, in particular for textured SMAs. An issue could be to use the general theory of tensor-representation proposed by Boehler to describe anisotropic behaviour of continuous media [48]. To date, macroscopic models assume isotropic SMAs. In this case, $f$ is reduced to a function of the three principal stresses $\sigma_{1}, \sigma_{2}$ and $\sigma_{3}$ or of the three invariants of the stress tensor $I_{\sigma}, \overline{I I}_{s}$ and $\overline{I I}_{s}$ defined as :

$$
I_{\sigma}=\operatorname{tr}(\underline{\underline{\mathbf{\sigma}}})=\sigma_{1}+\sigma_{3}+\sigma_{3} \quad ; \overline{I I}_{s}=\frac{1}{2} \operatorname{tr}(\underline{\underline{\underline{s}}} \cdot \underline{\underline{\mathbf{s}}}) ; \quad \overline{I I I}_{s}=\frac{1}{3} \operatorname{tr}(\underline{\underline{\mathbf{s}} . \underline{\underline{s}} . \underline{\underline{\mathbf{s}}})}) \quad \text { where } \underline{\underline{\mathbf{s}}}=\underline{\underline{\mathbf{\sigma}}}-\frac{I_{\sigma}}{3} \underline{\underline{\mathbf{I}}}
$$


where $\underline{\underline{\mathbf{s}}}$ is the deviatoric stress tensor. In our presentation we prefer to use the polar representation of $\underline{\boldsymbol{\sigma}}$, which involves the hydrostatic pressure, $p$, the deviatoric stress tensor intensity, $Q_{\sigma}$, and the phase of the deviatoric stress tensor, $\varphi_{\sigma}$, defined as :

$$
p=-\frac{I_{\sigma}}{3} \quad ; \quad Q_{\sigma}=\sqrt{2 \overline{I I}_{s}} \quad ; \quad \cos 3 \varphi_{\sigma}=\frac{3 \sqrt{6} \overline{I I}_{s}}{Q_{\sigma}{ }^{3}}
$$

The meanings of $Q_{\sigma}$ and $\varphi_{\sigma}$ are illustrated in Figure 8(a) in the deviatoric stress plane.

Many 3D-models have been initially developed assuming a Von Mises transformation surface, i.e.

$$
f=Q_{\sigma}-\sqrt{2} \tau_{t r}=0
$$

where $\tau_{\pi}$ is the temperature-dependent transformation shear stress. The results presented in section 3 demonstrate that this criterion, independent of $I_{\sigma}$ and $\varphi_{\sigma}$, cannot satisfactorily model transformation/reorientation yield surface in SMAs. The role of pressure (or $l_{\sigma}$ ) on superelastic behaviour has been studied experimentally only in the case of textured SMAs [27-28]. In that respect, as transformation/reorientation occur with a quasi-nil volume variation, all the developed macroscopic models assume that the pressure effect in isotropic SMAs is negligible. Hence, to account for results summarised in section 3 , the critical function $f$ has to be dependent on the stress phase $\varphi_{\sigma}$. Several forms of $f$ have been proposed in the theory of plasticity [50-52]. Compared to the usual Von Mises criterion, the form initially adopted by Patoor et al [46] requires only one additional constitutive parameter $\gamma_{0}$ :

$$
f=Q_{\sigma}-\frac{\sqrt{2} \tau_{t r}}{1+\gamma_{o} \cos 3 \varphi_{\sigma}}=0
$$

Such a yield surface was fitted with combined tension-compression-torsion experiments performed on a polycrystalline NiTi by Lexcellent et al [26] (cf. Figure 7(b)).

Orgéas and Favier [14] proposed to use an improved yield surface determined by Stutz [52], which involves a second constitutive constant parameter, $n_{o}$ :

$$
f=Q_{\sigma}-\frac{\sqrt{2} \tau_{t r}}{\left[1+\gamma_{o} \cos 3 \varphi_{\sigma}\right]^{n_{o}}}=0
$$

Figure 8(b) show that such kind of yield surface fits well the experimentally measured transformation stresses at different testing temperatures for an isotropic polycrystalline $\mathrm{NiTi}$ in shear, tension and compression. This yield surface requires only two additional parameters compared to Von Mises criterion $\left(\gamma_{0}=0.9\right.$ and $\left.n_{o}=0.1\right)$.

(a)

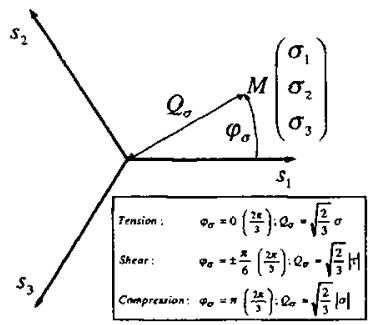

(b)

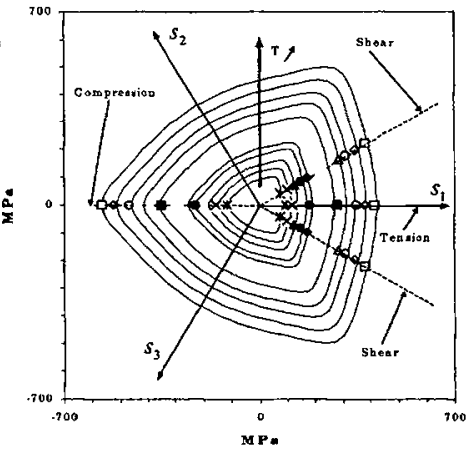

Figure 8 : (a) Polar representation of the stress tensor in the deviatoric stress plane, (b) experimental shear, tension and compression results obtained on a superelastic NiTi polycrystal at different temperatures above $\mathbf{M}_{\mathbf{s}}$ and identification of the yield criterion (5) proposed in [14] $\left(\gamma_{0}=0.9\right.$ and $\left.n_{o}==0.1\right)$.

\section{CONCLUSION}

Experiments other than tension tests on SMAs provide further information that enhance the understanding of the martensitic transformation and martensite reorientation. This effort has to be pursued in both 
experimental and theoretical studies. This will allow the development of improved constitutive relationships to model stress-train behaviour of isotropic and textured SMAs.

\section{References}

1. Rozner A.G., Wasilewski, J.Inst.Metals, 94, 169, (1966).

2. Melton K.N. and Mercier O., Acta Met., 29, 393, (1980).

3. Miyazaki S, Otsuka K.,Suzuki Y., Scripta Met, 15, 287, (1981).

4. SaburiT., Yoshida M., Nenno S., Scripta Met, 18, 363, (1984).

5. Tobushi H., Tanaka K., Hori T., Sawada T., Hattori T., JSME Int. Jal, A, 36, 3, 314 (1993).

6. Ford D.S. and White S.R., Acta Met., 44, 2295, (1996).

7. Liu Yinong, Liu Yong and Van Humbeeck J., Scripta Mat, 39, 8, 1047 (1998).

8. Miyazaki S., Imai T.,Otsuka K.,Suzuki Y. Scripta Met, 15, 853 (1981).

9. Shaw J.A. and Kyriakides S., J. of Mech. Phys. Solids, 43, 8, 1243,(1995).

10. Liu Yinong and Xiang Hong, J. of Alloys and Compounds, 270, 154, (1999).

11. Warlimont H., Delaey L., Krishnan R.V., Tas H., J. Mater. Sci, 9, 1545, (1974).

12. Liu Yinong, Mat. Science and Eng., A271, 506, (1999).

13. Orgéas L. and Favier D., J. de Phys IV, 5, 605, (1995).

14. Orgéas L. and Favier D., Acta Mater., 46, 15, 5579, (1998).

15. Manach P.Y. and Favier D., Mat. Sci. Eng., A222, 45, (1997).

16. Sehitoglu H., Karaman I., Anderson R., Zhang X., Gall K., Maier H.J., Chumlyakov Y. Acta Mater., 48, 3311, (2000).

17. Favier D., Habilitation Thesis, INPG, Grenoble, France, (1988).

18. Tourabi A., Wack B., Favier D., Mat. Science Forum, 56-58, 535, (1990).

19. Vacher P. and Lexcellent C, Proc. of Mech. Behaviour of Ma. VI, Perg. Press, 231, (1991).

20. Liu Yinong, Houver I., Xiang H., Bataillard H., Miyazaki S., Met. and Mat. Trans. A, 30A, 1275, (1999).

21. Fischer F.D., Oberaigner E.R., Reisner G., Sun Q.P., Tanaka K., Rev. Eur. des E.F., 7, 8, 9, (1998).

22. Shaw J.A., Int. J. of Plasticity, 16, 541,(2000).

23. Orgeas L. and Favier D., Rev. Eur. des E.F., 7, 8, 111, (1998).

24. Wazilewski R.J., Met. Trans., 2, 2973, (1971).

25. Roumagnac P., Ph. D. Thesis, UTC, Compiègnes, France, (1993).

26. Lexcellent C., Rejzner J., Bouvet C., Robinet P., Calloch S., Proc. of $38^{\text {th }}$ Int. Conf. Of Met., Québec, Canada, 73 (1999).

27. Jacobus K., Sehitoglu H., Balzer M., Met.and Mat.Trans., Vol. 27A, 3066 (1996).

28. Gall K.,Sehitoglu H., Maier H.J., Jacobus K., Met.and Mat.Trans., 29A, 765 (1998).

29. Otsuka M., Shimizu K., Int. Metals Rev., 31, 3, 93, (1986).

30. Sakamoto H., Tanigawa M., Otsuka M., Shimizu K., Proc. of ICOMAT 79, Cambridge, Massachusetts, USA, 633, (1979).

31. Plietsch R. and Ehrlich K., Acta Mat., 45, 2417, (1997).

32. Xie Z.L., Liu Yong, Van Humbeeck J., Delaey L., Acta Mater., 46, 12, 4325, (1998).

33. Adler P.H., Yu W., Pelton A.R., Zadno R., Duerig T.W. and Baresi R., Scripta Met., 24, 943, (1990).

34. Rogueda C., Lexcellent C., Bochet L., Arch. Mech., 48, 1025, (1996).

35. Sitner P., Hara Y., Tokuda M., Met. and Mat. Trans. A, 26A, , 2923, (1995).

36. Patoor E., Eberhardt A., Berveiller M., $8^{\text {th }}$ Int. Symp. Metall. Mat. Sci.,Roskilde,Denmark,465,(1987).

37. Caneiro A., Chandrasekaran M. Scripta Mat, 22, 1047, (1988).

38. Ono N. and Shimanuki H., Scripta Met, 24, 2269 (1990).

39. Tas H., Delaey L., Deruyttere A., Z. Metallkd., 64, 855, (1973).

40. Otsuka K., Wayman C.M., Nakai K., Sakamoto H., Shimizu K., Acta Metall, 22, 1475, (1976).

41. Miyazaki S., Kimura S., Otsuka K., Suzuki Y., Scripta Metall., 18, 883, (1984).

42. Saburi T., Nenno S., Proc. Int. Conf. on Solid-Solid Phase Transformation, 1455, (1981).

43. Buchheit T.E., Kumpf S.L., Wert J., Acta Met., 11, 4189, (1995).

44. Buchheit and Wert J., Met. Trans., 27A, 269, (1996).

45. Liu Yong, Xie Z.L.,Van Humbeeck J., Delaey L., Acta Mater., 47, 2, 645, (1999).

46. Patoor E., El Amrani M., Eberhardt A. and Berveiller M., J. de Phys. IV, 5, 495, (1995).

47. Gall K., Sehitoglu H.,Int. J.of Plasticity, 15, 69, (1999).

48. Boehler J.P., J. de Mécanique, 17, 2, 153, (1978).

49. Kakeshita T., Shimizu K., Nakamichi S., Tanaka R., Endo S., Ono F., Met.Trans. Jim, 33,1,1, (1992).

50. Krenk S., J. of Eng. Mech., 201, (1996).

51. Haythornthwaite R.M., Mech. Res. Comm., 12, 2, 87, (1985).

52. Stutz P., Habilitation Thesis, Université Joseph Fourier, Grenoble, France, (1979). 